\title{
Three-dimensional Path Planning for Underwater Vehicles Based on an Improved Ant Colony Optimization Algorithm
}

\author{
L.Yang ${ }^{1, *}$, K.S.Li ${ }^{1}$, W.S.Zhang ${ }^{2}$, Y.Wang ${ }^{3}$, Y.Chen ${ }^{1}$ and L.F.Zheng ${ }^{1}$ \\ ${ }^{1}$ College of Mathematics and Informatics, South China Agricultural University, Guangzhou, 510642, China \\ ${ }^{2}$ Institute of Automation, Chinese Academy of Sciences, Beijing, 100190, China \\ ${ }^{3}$ Department of Computer science and information systems, Bradley University, Peoria, Illinois, 61625, USA
}

Received 20 October 2015; Accepted 13 December 2015

\begin{abstract}
Three-dimensional path planning for underwater vehicles is an important problem that focuses on optimizing the route with consideration of various constraints in a complex underwater environment. In this paper, an improved ant colony optimization (IACO) algorithm based on pheromone exclusion is proposed to solve the underwater vehicle 3D path planning problem. The IACO algorithm can balance the tasks of exploration and development in the ant search path, and enable the ants in the search process to explore initially and develop subsequently. Then, the underwater vehicle can find the safe path by connecting the chosen nodes of the $3 \mathrm{D}$ mesh while avoiding the threat area. This new approach can overcome common disadvantages of the basic ant colony algorithm, such as falling into local extremum, poor quality, and low accuracy. Experimental comparative results demonstrate that this proposed IACO method is more effective and feasible in underwater vehicle 3D path planning than the basic ACO model.
\end{abstract}

Keywords: Ant colony algorithm, Pheromone exclusion, 3D path planning, Environment modeling

\section{Introduction}

Three-dimensional (3D) path-planning problem requires finding a collision-free and optimal path for an underwater vehicle from start point to goal point in a complex underwater environment. Many studies on path-planning algorithms have been conducted, but most of these methods are for the 2D space [1], [2], [3], [4]. The algorithm of 3D path planning is difficult because of its complicated calculation process, large amount of stored information, difficulty in directly performing global planning, and other issues. Numerous algorithms have been used to solve the 3D path-planning problem, such as artificial potential field [5], $\mathrm{A}^{*}$ [6], genetic [7], and particle swarm optimization [8] algorithms. Although these algorithms have contributed to the research on the path-planning problem in the 3D space, they have limitations. The potential field algorithm cannot avoid being trapped in local optimal path and cannot be extended directly when the optimization rule is complicated. The $A^{*}$ algorithm can be used to solve high-dimensional problems, but as the dimension increases, the space-time requirement of this algorithm becomes difficult to meet. The genetic algorithm can accomplish the planning when the environment condition is simple, but it has difficulty finding a feasible path in a complex environment.

Ant colony optimization (ACO) [9] algorithm proposed by $\mathrm{M}$. Dorigo in 1991 is a new intelligent optimization algorithm [10], [11]. As a bionic algorithm, ACO has many characteristics, such as distributed computing, positive

* E-mail address: yanglei_s@scau.edu.cn ISSN: 1791-2377 @ 2015 Kavala Institute of Technology. All rights reserved. feedback mechanism, and swarm intelligence [12]. It can be used in $2 \mathrm{D}$ or $3 \mathrm{D}$ path planning, but the conventional $\mathrm{ACO}$ easily falls into local extremum, and has poor quality and low accuracy in the $3 \mathrm{D}$ path planning. In this paper, the path-planning problem of underwater vehicles in $3 \mathrm{D}$ space based on ACO is studied, and an improved ACO (IACO) search algorithm based on pheromone exclusion and $3 \mathrm{D}$ space environment modeling method is proposed. The IACO algorithm is based on the behavior of real ants. As ants move, the pheromones they release in their search path includes not only an attractive part but also an exclusive part. The IACO algorithm enables the ants in the search path process to explore initially and to develop subsequently. This algorithm has been applied to path planning in $3 \mathrm{D}$ space underwater. The simulation results show that the IACO algorithm has good robustness and high efficiency, can overcome the shortcomings of the conventional ACO, and can effectively improve the quality and precision of the search path. Moreover, the stability of the output is better than that of the conventional ACO.

\section{3D space environment mathematical modeling}

Environment modeling expresses real 3D space environment information in an abstract pattern, which is closely related to the optimization algorithm, and influences the efficiency of the path planning directly [13]. The environment abstract modeling method is described as follows: first, the top left corner of the 3D map vertex is set as the origin of the coordinates of 3D space $A$; in point $A$, the $3 \mathrm{D}$ coordinate system is established. The $x$-axis follows the longitude degree increment direction and passes point $A$ in the water level. The $y$-axis follows the latitude degree increment 
direction and passes point $A$ in the water level. The $z$-axis is perpendicular to the water level, which passes point $A$ and moves upward to the water level. In the Cartesian coordinate system, $A-x y z$ takes point $A$ as a vertex, taking the maximum length $|\mathrm{AB}|$ along the axis $x$, taking the maximum length $|\mathrm{AD}|$ along the axis $y$, and taking the maximum length $\mid \mathrm{AA}$ ' along the axis $z$. Then, a cube space ABCD-A'B'C'D' can be obtained, which is the $3 \mathrm{D}$ planning space [14], as shown in Figure 1.

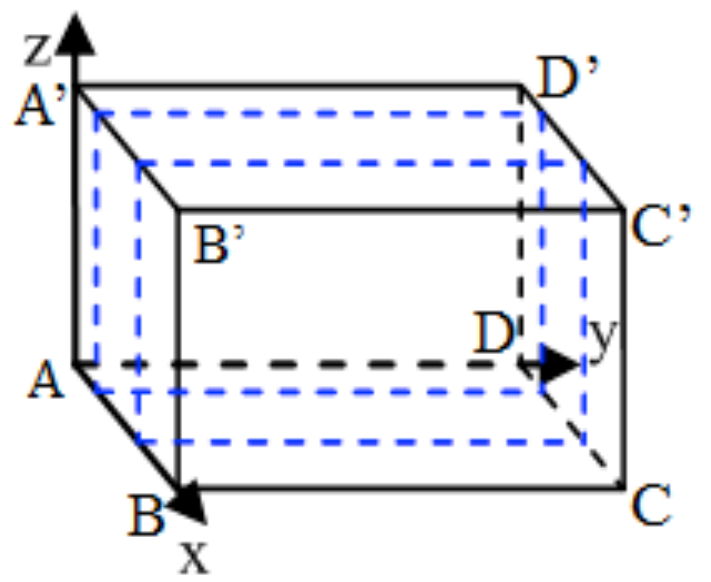

Fig. 1. Three-dimensional model

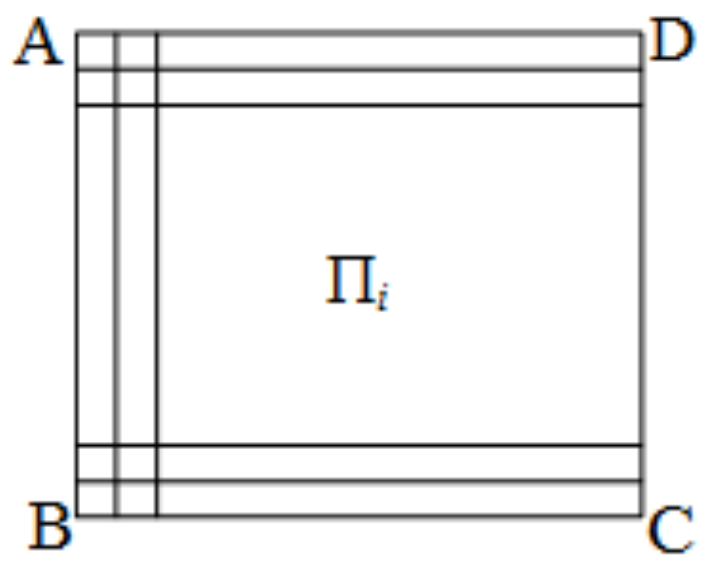

Fig. 2. Planar graph partition

After establishing the planning space, we have to further divide the space and extract the 3D path planning of grid points to obtain the abstract environment model. First, we plot the planning space $\mathrm{ABCD}-\mathrm{A}^{\prime} \mathrm{B}$ ' $\mathrm{C}$ ' $\mathrm{D}$ ' into $n$ parts along line $\mathrm{AB}$ to obtain $n+1$ planes $\Pi_{i}(i=0,1,2, \cdots, n)$. Then, we plot arbitrary plane $\Pi_{i}$ along $\mathrm{AD}$ into $m$ parts and along AA' into $l$ parts, and we have to solve the intersection between planes for path planning of the grid points. The plane is divided, as shown in Figure 2.

Through the aforementioned steps, the planning space ABCD-A'B'C'D' is dispersed into several 3D points. With $P^{*}$ as the aggregate of these points in this paper, any point $a$ in the aggregate corresponds to two coordinates, namely, sequence number coordinate $a^{1}(i, j, k)(i=0,1,2, \cdots, n, j=0,1$, $2, \cdots, m, k=0,1,2, \cdots, l)$ and position coordinate $a^{2}\left(x_{i}, y_{i}, z_{i}\right)$. The sequence number coordinate $a^{1}(i, j, k)$ is the sequence number of $a$ along the three directions in the process of plotting the planning space. As shown in Figure $1, i, j$, and $k$ are the sequence numbers of point $a$ along lines $\mathrm{AB}, \mathrm{AD}$, and AA'. The position coordinate $a^{2}\left(x_{i}, y_{i}, z_{i}\right)$ of point $a$ is longitude excursion distance, latitude excursion distance, and depth excursion distance of the position corresponding to point $A$. The IACO based on pheromone exclusion principle is used in this $3 \mathrm{D}$ path point planning to establish an optimal path between the starting point and the target point according to certain criteria.

\section{Improved ant colony algorithm with pheromone exclusion for underwater vehicle 3D path planning}

\subsection{Representation of pheromones}

The pheromone is a carrier of information from the past; it directly affects the global convergence and computational efficiency of the ant colony algorithm. In the path planning problem, we usually take the path between the adjacent nodes as the pheromone carrier, but this method applies only to small-scale problems [15]. An environment model structure map of the $3 \mathrm{D}$ path-planning problem has more nodes. If we take the path between the adjacent discrete nodes as the pheromone carrier, then the space complexity of the algorithm becomes unbearable. Position setting and updated method of pheromone are crucial for the ant colony algorithm to search $3 \mathrm{D}$ paths. The mathematical model of the $3 \mathrm{D}$ environment has divided the entire search space into a series of discrete points; the discrete points are the nodes that the ant colony algorithm needs to search for. In this study, the pheromone released by the ant in the search path includes not only the attractive part but also the exclusive part. It makes the ants in the search path process tend to explore initially, and tend to develop in the later search. The pheromone is stored in the discrete points in the $3 \mathrm{D}$ environment mathematical model. Each discrete point is stored with the pheromone value. The pheromone value represents the ant's attraction or repulsion level, as each ant maintains its own pheromone. The pheromone value of the discrete node has to be updated locally after each ant passes by. When all the ants have set up the path, the pheromone is updated globally [16].

\subsection{Design of heuristic function}

Heuristic function is an important component of the path planning algorithm in 3D space. This function calculates the $3 \mathrm{D}$ path selection probability of each point within the visible area for the ants searching from the current point to the next point. The heuristic function is not only the carrier of characterization of future information but is also an important part of the $3 \mathrm{D}$ route planning algorithm. Experimental results show that the heuristic function must ensure that the ant colony algorithm searches the global optimal solution within a reasonable period of time. The present study adopts the following heuristic function:

$$
H(i, j, k)=D(i, j, k)^{w_{1}} \cdot S(i, j, k)^{w_{2}} \cdot Q(i, j, k)^{w_{3}}
$$

where the factor $D(i, j, k)$ is the path length from the current node to the next node to allow the planning path to be as short as possible. We use the function value of $S(i, j, k)$ to represent the safety grade of the feasible point $(i, j, k)$. The value is 0 if the points cannot choose, and it makes the ant select a safe point. The factor $Q(i, j, k)$ is the path length of the next node to the target node. It makes the ants choose a 
distance closer to the target; $w_{1}, w_{2}, w_{3}$ are the coefficients that represent the relative important grades of the mentioned factors.

The factor $D(i, j, k)$ is computed as follows:

$$
D(i, j, k)=\operatorname{sqrt}\left[\left(x_{a}-x_{b}\right)^{2}+\left(y_{a}-y_{b}\right)^{2}+\left(z_{a}-z_{b}\right)^{2}\right]
$$

where $a$ is the current node and $b$ is the next node.

The calculation formula of the factor $S(i, j, k)$ is as follows:

$$
S(i, j, k)=\frac{N u m-U N u m}{N u m}
$$

where Num is the number of feasible points in the visual domain space of point $(i, j, k)$, and UNum is the number of unfeasible points in the visual domain space.

The factor $Q(i, j, k)$ is computed as follows:

$$
Q(i, j, k)=\operatorname{sqrt}\left[\left(x_{b}-x_{d}\right)^{2}+\left(y_{b}-y_{d}\right)^{2}+\left(z_{b}-z_{d}\right)^{2}\right]
$$

where $b$ is the next node and $d$ is the target node.

\subsection{Design of ant colony search strategy}

\subsubsection{Visual domain space}

In this study, we choose the $x$-axis as the main direction of the $3 \mathrm{D}$ path planning. An underwater vehicle moves along the $x$-direction. To reduce the complexity of path planning, we simplify the vehicle movements through three operations: forward, transverse, and longitudinal movement. When the vehicle moves ahead one unit $L_{x, \max }$, we allow it to take the maximum lateral movement $L_{y, \max }$ and take the maximum longitudinal movement range $L_{z, \max }$. Thus, when the ant lies at $H(i, j, k)$ point on plane $\Pi_{i}$, a visual domain space of point $H(i, j, k)$ exists.

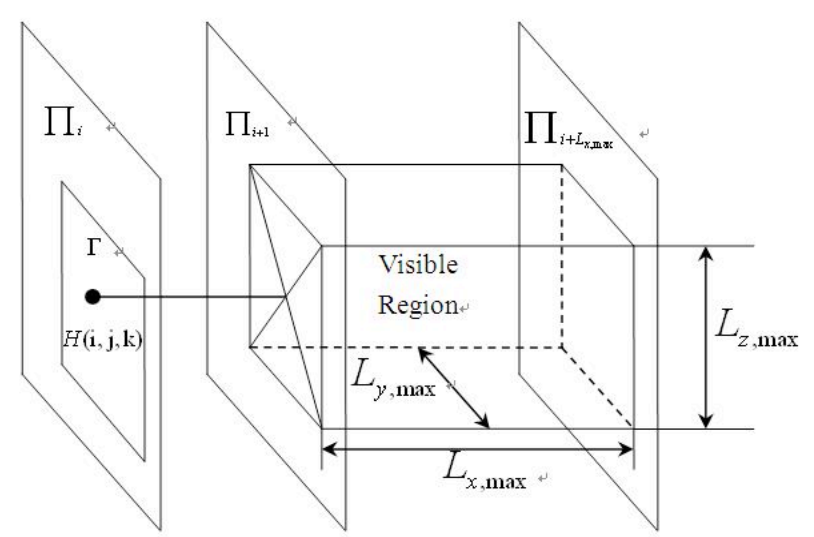

Fig. 3. Visual domain space

First, we provide the definition of the ant's visual domain space.

Definition 1 Visual domain space Given $L_{x, \text { max }}\left(L_{x, \text { max }} \in\{1,2, \cdots, n\}\right), L_{y, \text { max }}\left(L_{y, \text { max }} \in\{1,2, \cdots, m\}\right)$, and $L_{z \text {,max }}\left(L_{z, \text { max }} \in\{1,2, \cdots, l\}\right)$, as shown in Figure 3, we suppose that $\Gamma$ is the selection domain in the rectangular plane $\Pi_{i}$ that contains the ant's current path node, and $H(i, j, k)$ is the arbitrary feasible point in the space $\Gamma$.Thus, the rectangular

point sets $\quad\left(x, y, z \mid x \in\left\{i+1, i+2, \cdots, \min \left(n, i+L_{x, \max }\right)\right\}\right.$, $y \in\left\{\max \left(j-L_{y, \max } / 2,0\right), \cdots, j, \cdots \min \left(j+L_{y, \max } / 2, m\right)\right\}$, $\left.z \in\left\{\max \left(k-L_{z, \max } / 2,0\right), \cdots, k, \cdots \min \left(k+L_{z, \max } / 2, l\right)\right\}\right)$ can be called the visual domain space of point $H(i, j, k)$ [17].

Therefore, when the ant moves from the current node to the next node, the search area of the ant is limited to the visual domain space, which can simplify the search space and improve the search efficiency of the ant colony algorithm.

\subsubsection{Design of path search strategy}

In the ant colony algorithm, the path search is conducted according to the probability, and the ants use different decision rule, which is called pseudo-random proportional rule. This rule can use the distance heuristic information between the nodes as well as pheromone prior knowledge that is already stored, and can have a propensity to explore. The following process shows how ants in the present node $P_{i}$ in plane $\Pi_{i}$ choose the next path node $P_{i+1}$ in plane $\Pi_{i+1}$.

(1) The feasible path nodes in the selection space of plane $\Pi_{i+1}$ are chosen according to the abstract environment model.

(2) The heuristic information value $H_{i+1, u, v}$ of an arbitrary point $(i+1, u, v)$ in the selection space of plane $\prod_{i+1}$ is calculated.

(3) A new method of transfer probability calculation is proposed in this paper. The pheromone of ants in the path searching process contains both the attractive part and the exclusive part. The ant is attracted by its own released pheromones and is excluded by pheromones released by the other ants. We defined that a representation attracts weight $\Lambda_{i i+1}^{k}$ and a rejection of the weight $\Phi_{i i+1}^{k}$ for a feasible next node $P_{i+1}$ of the ant $k . \Lambda_{i i+1}^{k}$ is designed according to this standard, as shown in the following equation:

$$
\Lambda_{i i+1}^{k}(t)=\frac{\tau_{i i+1}^{k}(t)}{\sum_{j \in N_{i}^{k}(t)} \tau_{i j}^{k}(t)}
$$

The equation $\tau_{i i+1}^{k}(t)$ represents the value of pheromones released by the ant $k$ in the next path node $P_{i+1}$ in plane $\Pi_{i+1} \cdot \Lambda_{i i+1}^{k}$ is calculated through the amount of attracted pheromones of all subsequent feasible nodes corresponding to the node $P_{i}$ of ant $k$, and is standardized.

$\Phi_{i i+1}^{k}$ is designed according to this standard and is shown in the following equation:

$$
\Phi_{i i+1}^{k}(t)=\frac{\prod_{i i+1}^{k}(t)}{\sum_{j \in N_{i}^{k}(t)} \prod_{i j}^{k}(t)}
$$

where

$$
\prod_{i i+1}^{k}(t)=\sum_{\substack{m=1, \cdots, n_{k} \\(m \neq k)}} \tau_{i i+1}^{m}(t)
$$

Equation (7) represents the value of pheromones released by all other ants, except $k$ in the next path node $P_{i+1}$ in 
plane $\prod_{i+1} \cdot \Phi_{i i+1}^{k}$ is calculated by the amount of excluded pheromones of all subsequent feasible nodes corresponding to the node $P_{i}$ of ant $k$, and is standardized.

According to the preceding definition, the pheromone weight of the arbitrary feasible point in plane $\prod_{i+1}$ is defined as

$$
\eta_{i i+1}^{k}(t)=\frac{\left(\Lambda_{i i+1}^{k}(t) / \Phi_{i i+1}^{k}(t)\right)^{\alpha}}{\sum_{i i+1 \in N_{i}^{k}(t)}\left(\Lambda_{i i+1}^{k}(t) / \Phi_{i i+1}^{k}(t)\right)^{\alpha}}
$$

Where the ratio of attraction and exclusion balances the relationship between exploration and development. Thus, the ants in the search path process tend to explore initially and to develop subsequently.

The next path node $P_{i+1}$, which has a position coordinate of $P\left(x_{i+1}, y_{i+1}, z_{i+1}\right)$, was chosen in plane $\prod_{i+1}$ according to the following pseudorandom proportional rule:

$$
p_{i i+1}^{k}(t)= \begin{cases}\frac{\eta_{i i+1}^{k}(t) H\left(x_{i+1}, y_{i+1}, z_{i+1}\right)}{\sum_{i i+1 \in N_{i}^{k}(t)} \eta_{i i+1}^{k}(t) H\left(x_{i+1}, y_{i+1}, z_{i+1}\right)} & \text { feasible point } \\ 0 & \text { else }\end{cases}
$$

where $\eta_{i i+1}^{k}(t)$ is the pheromone amount weight saved in node $P_{i+1}$ in plane $\Pi_{i+1}$.

4) Roulette algorithm is selected to choose the next path node in plane $\prod_{i+1}$, according to the probability of each point.

\subsection{Pheromone update rule}

Pheromone update consists of two parts, namely, local pheromone trail update and global pheromone trail update [18]. The local pheromone trail is updated after the ant passes the point. The pheromone of the point decreases. The pheromone update can increase the probability of the point that the ant has not searched and achieve the goal of global search. The update method is expressed as follows:

$$
\tau_{i j k}=(1-\zeta) \tau_{i j k}
$$

where $\tau_{i j k}$ is the pheromone value of the feasible point $(i, j, k)$ and $\zeta$ is the attenuation coefficient of pheromones.

Unlike the conventional ACO algorithm, the IACO algorithm allows each ant to maintain its own pheromone. Thus, the pheromone of each ant also volatilized independently. The local update method of the IACO algorithm is shown as follows:

$$
\tau_{i j k}^{k}(t+1)=\tau_{i j k}^{k}(t)+Q^{k}
$$

where $Q^{k}$ is the fixed size amount of the pheromone and is a positive constant. After a complete path was established, the pheromone was volatilized as follows:

$$
\tau_{i j k}^{k}(t)=(1-\rho) \tau_{i j k}^{k}(t)
$$

where $\rho(0<\rho<1)$ is a parameter.

The global pheromone trail is updated after the ant has constructed its path. We choose the shortest path based on the path length from the set of paths as standard, and we increase the pheromone value of each node. The global pheromone update method of the conventional ACO algorithm is shown as follows: $\tau_{i j k}=(1-\rho) \tau_{i j k}+\rho \Delta \tau_{i j k}$

$\Delta \tau_{i j k}=\frac{K}{\min (\text { length }(m))}$

where length $(m)$ is the length of the No. $m$ ant's path, $\rho(0<\rho<1)$ is a parameter, and $K$ is the coefficient.

The global pheromone trail of the IACO algorithm is updated after all ants have constructed their path. The update method of the IACO algorithm is shown as follows:

$\tau_{i j k}^{k}(t+1)=\tau_{i j k}^{k}(t)+\frac{Q^{k}}{f\left(x^{k}(t)\right)}$

where $f\left(x^{k}(t)\right)$ is the best fitness value in this study, which indicates the cost of the No. $k$ ant to construct its path. The global pheromone trail is updated only for the iterative optimal or global optimal path in the IACO algorithm.

\subsection{Process design of algorithm}

In this paper, the algorithm of the $3 \mathrm{D}$ path planning process for an underwater vehicle is described as follows:

Step 1. After the model of the abstract 3D environment has been built, and the starting point and target position in abstract environment model has been determined, the main direction of the ant movement is also determined. Then, all the ants are placed in the starting point. The parameters needed to be set in the algorithm begin to be initialized.

Step 2. On the basis of heuristic information and pheromone weight value, the next point of the ant searching is determined according to formulas (1), (8), and (9).

Step 3. The local pheromone trail is updated according to formulas (11) and (12).

Step 4. We determine whether all the ants completed building a path. If they did not, then we return to Step 2.

Step 5. The global pheromone trail is updated according to formula (15) to determine whether the algorithm satisfies the stop condition and the optimal result output meets the conditions. Otherwise, we return to Step 2.

Figure 4 shows the flowchart of the improved ACO algorithm for underwater vehicle 3D path planning.

\section{Simulations}

In this section, we show the performance of the IACO algorithm as it is applied to the 3D path planning problems, and we compare the performance of this algorithm with that of the conventional ACO algorithm. We use MATLAB for the simulation. The simulation is based on real underwater terrain data. We divide the $21 \mathrm{~km} * 21 \mathrm{~km} * 2 \mathrm{~km}$ underwater region by means of uniform mesh method, and we use the IACO algorithm to search the collision-free path from the starting point to the target point of this region. To facilitate problem solving, we set the deepest point in this area to zero. The height of the other point according to the height difference of the deepest point is obtained in turn. 
The 3D simulation diagram for the water area of the model is shown in Figure 5.

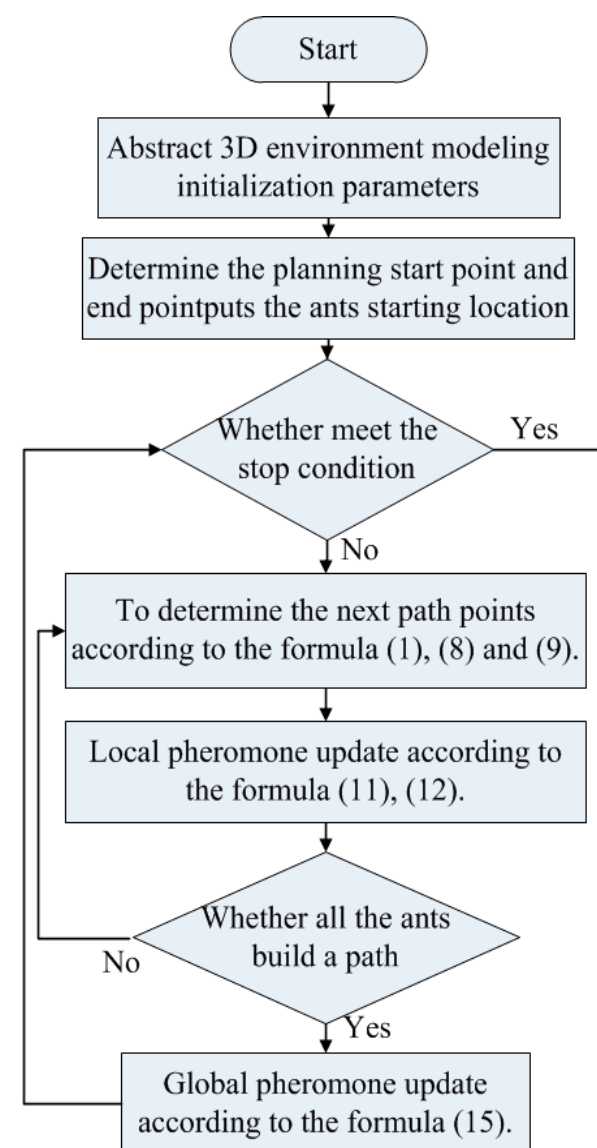

Fig. 4. Flowchart of improved ACO algorithm

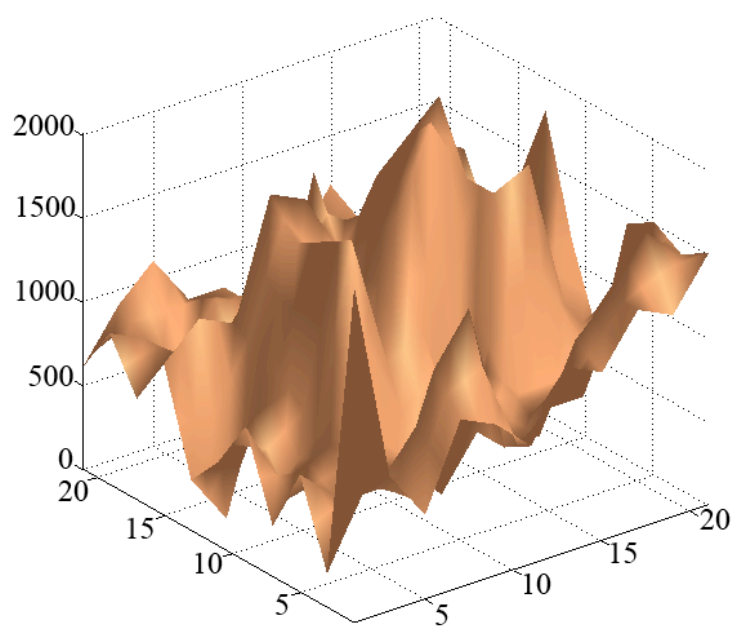

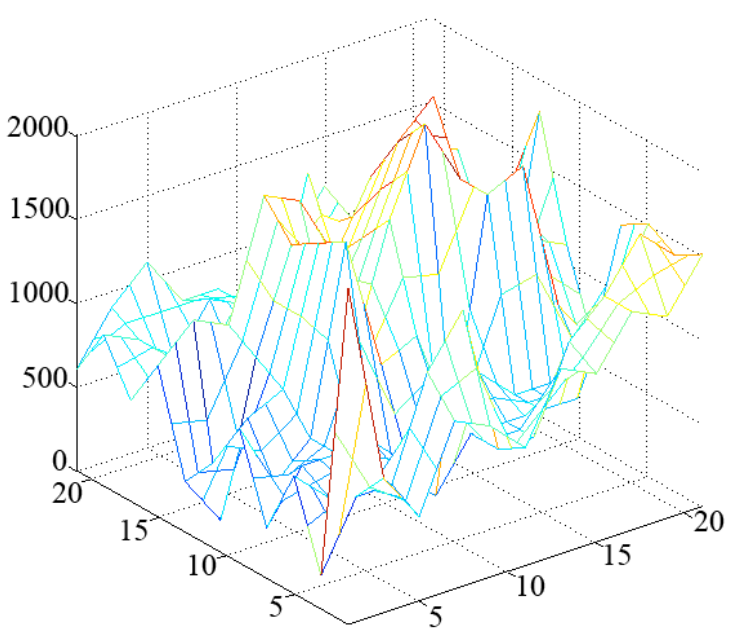

Fig. 5. 3D simulation diagrams

\subsection{Simulation 1}

Simulation 1 is performed on a relatively flat plane of the environment with the set start point $(1,10,800)$ and goal point $(21,4,1000)$. We first use the conventional ant colony algorithm for path planning in the water, and then we use the IACO algorithm based on the pheromone exclusion principle for path optimization between the initial point and the target point. The parameter setting is shown in Table 1.

Table 1. Parameters of the algorithm

\begin{tabular}{cccccc}
\hline $\begin{array}{c}\mathrm{X} \text { axis grid } \\
\text { number }\end{array}$ & $\begin{array}{c}\mathrm{Y} \text { axis } \\
\text { grid } \\
\text { number }\end{array}$ & $\begin{array}{c}\mathrm{Z} \text { axis } \\
\text { grid } \\
\text { number }\end{array}$ & $\begin{array}{c}\text { ant } \\
\text { number }\end{array}$ & $\tau_{0}$ & $\zeta$ \\
\hline 21 & 21 & 10 & 10 & 1 & 0.2 \\
\hline $\begin{array}{c}\text { iteration } \\
\text { number }\end{array}$ & $\mathrm{K}$ & $\alpha$ & $Q^{k}$ & $f\left(x^{k}(t)\right.$ & $\rho$ \\
\hline 100 & 100 & 5 & 100 & $\begin{array}{c}\text { optimal } \\
\text { fitness }\end{array}$ & 0.5 \\
\hline
\end{tabular}

The experiment is conducted eight times. The planning path length of the conventional ACO and the IACO is shown in Table 2.

In Experiment 1, the optimal path length of ACO planning is $28.3183 \mathrm{~km}$, and the optimal path length of IACO planning is $25.1251 \mathrm{~km}$. The simulation comparison charts is as follows.

Table 2. Comparison of path length (unit: $\mathrm{km}$ )

\begin{tabular}{cccccccccc}
\hline & 1 time & 2 times & 3 times & 4 times & 5 times & 6 times & 7 times & 8 times & average \\
\hline ACO & 31.1514 & 32.2239 & 30.9962 & 34.4189 & 31.8780 & 31.7936 & 28.3183 & 31.9156 & 31.5869 \\
\hline IACO & 27.5471 & 28.7836 & 25.1251 & 27.9500 & 25.8855 & 27.1325 & 25.1399 & 25.8855 & 26.6811 \\
\hline
\end{tabular}




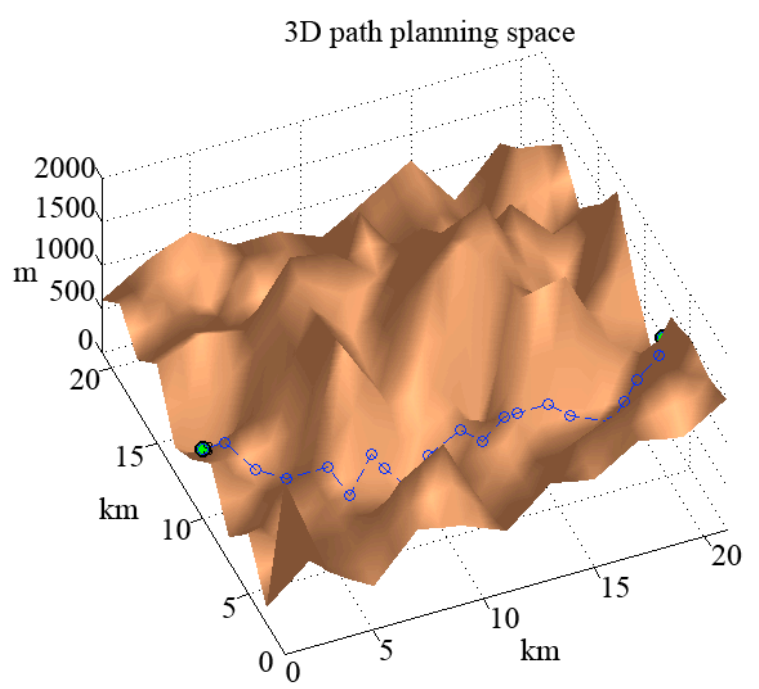

$3 \mathrm{D}$ path planning space

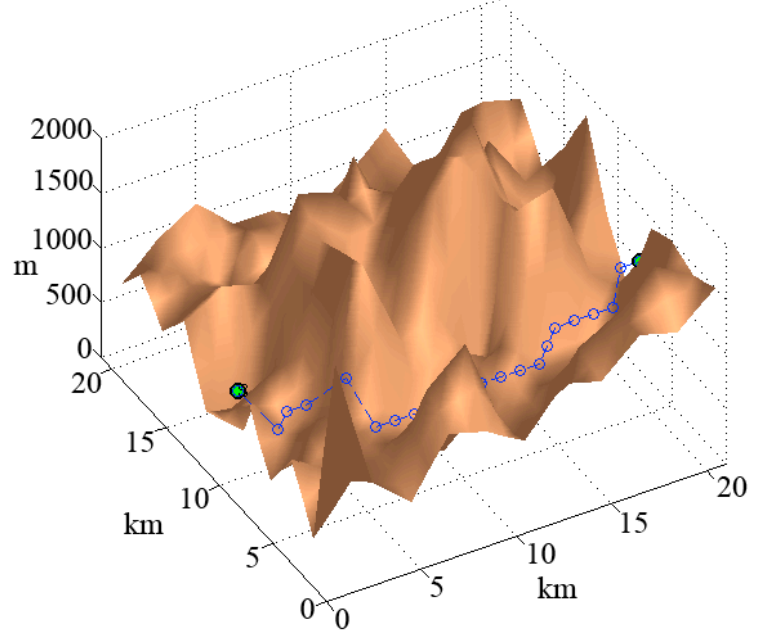

Fig. 6. Perspective diagram of the optimal path with ACO and IACO planning

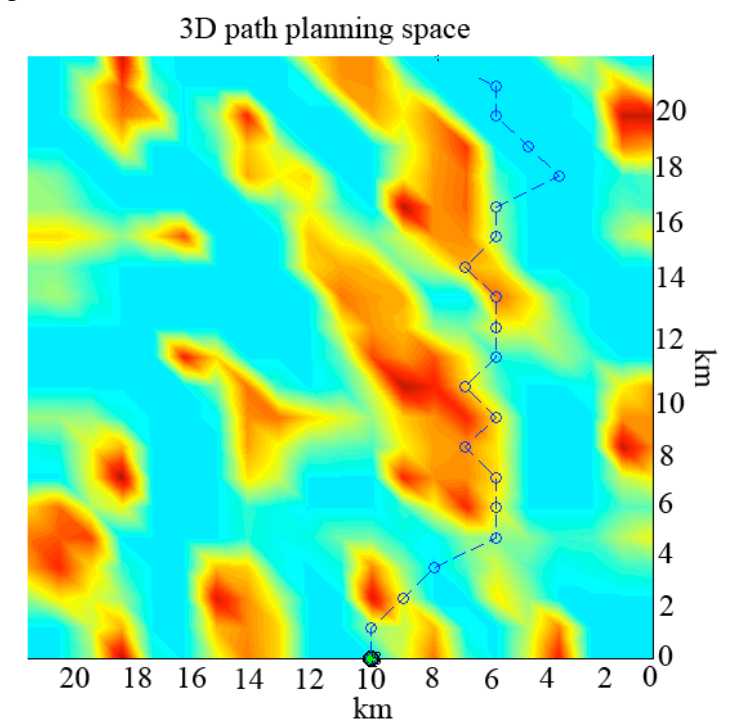

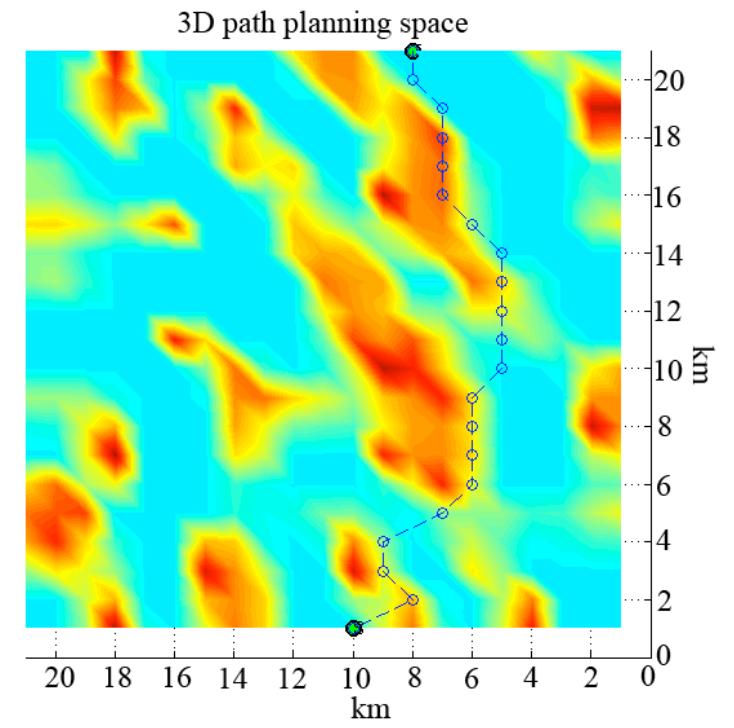

Fig. 7. Top view of the optimal path with ACO and IACO planning
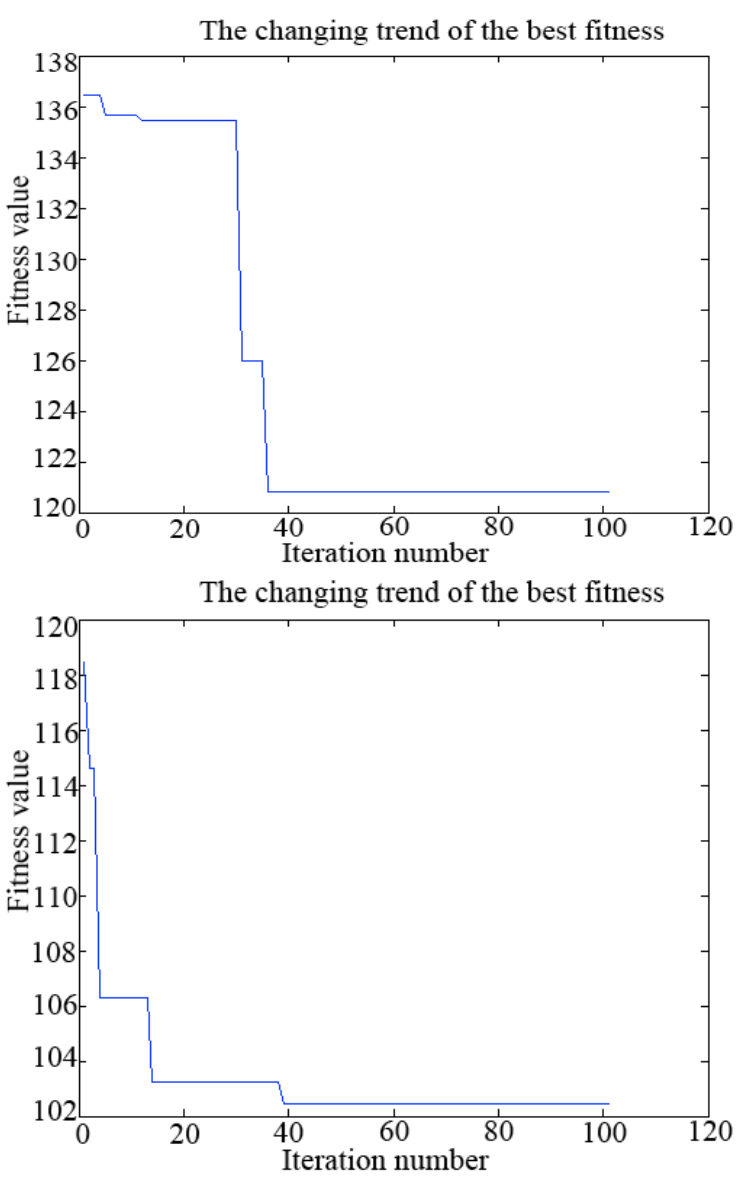

Fig. 8. Fitness variation diagram of ACO and IACO planning

In Experiment 1, the average path length of the ACO algorithm planning is $31.5869 \mathrm{~km}$, and the average path length of the IACO algorithm planning is $26.6811 \mathrm{~km}$. The optimal path time comparison table of the ACO and IACO algorithms is presented in Table 3.

Table 3. Optimal path time comparison table

\begin{tabular}{c|c}
\hline & E_time(s) \\
ACO & 1.805105 \\
IACO & 2.825599 \\
\hline
\end{tabular}


In Experiment 1, the simulation results of ACO planning show that the path can fluctuate easily in the local depth, whereas IACO performs better. The 3D average path length of the IACO planning is significantly shorter than that of the ACO. However, because of increased calculation of the weight of pheromone attraction and exclusion in IACO, this algorithm is slightly slower than ACO.

\subsection{Simulation 2}

Simulation 2 was performed on a relatively steep plane of the environment, with the set start point $(1,18,800)$ and the goal point $(21,16,1200)$. The values in Table 1 were used in the parameter setting of the algorithm simulation process. The experiment was conducted eight times. The planning path lengths of the ACO and IACO algorithms are shown in Table 4.

Table 4. Comparison of path length (unit: $\mathrm{km}$ )

\begin{tabular}{cccccccccc}
\hline & 1 time & 2 times & 3 times & 4 times & 5 times & 6 times & 7 times & 8 times & average \\
\hline ACO & 27.5298 & 28.2408 & 41.5395 & 29.4466 & 43.0707 & 30.2734 & 27.8154 & 27.7280 & 31.9555 \\
\hline IACO & 23.5443 & 24.3555 & 25.1605 & 23.9353 & 23.5047 & 24.7242 & 24.7299 & 23.9015 & 24.2319 \\
\hline
\end{tabular}

In Experiment 2, the optimal path length of ACO planning is $27.5298 \mathrm{~km}$, and the optimal path length of IACO planning is $23.5047 \mathrm{~km}$. The simulation comparison charts are shown in Figure 9, Figure 10 and Figure 11.
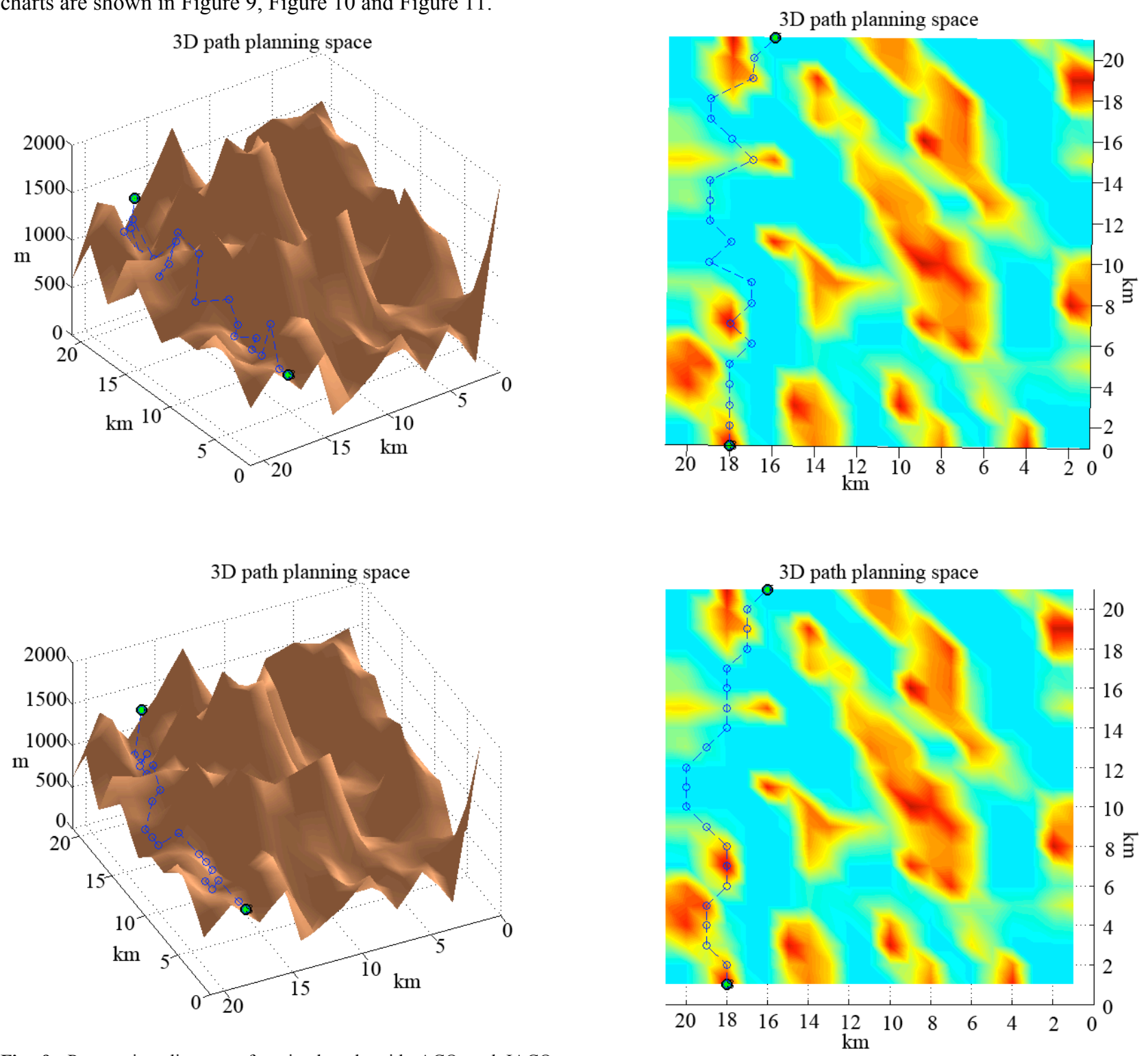

Fig. 9. Perspective diagram of optimal path with ACO and IACO planning

Fig. 10. Top view of optimal path with ACO and IACO planning 

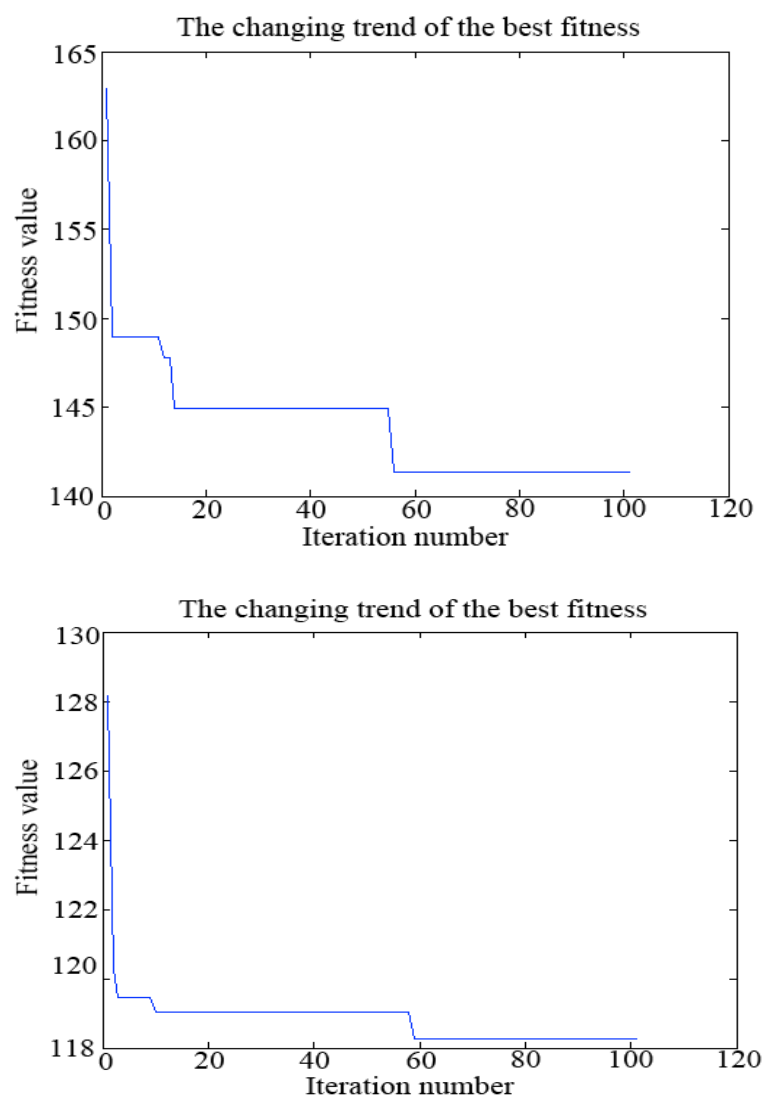

Fig. 11. Fitness variation diagram of $A C O$ and IACO planning
In this simulation, the optimal path time comparison of the ACO and IACO algorithms is shown in Table 5.

Table 5. Optimal path time comparison table

\begin{tabular}{l|l}
\hline & E_time(s) \\
ACO & 2.699045 \\
IACO & 4.097759 \\
\hline
\end{tabular}

In Experiment 2, the simulation results show that the 3D average path length of the IACO planning is significantly shorter than that of ACO in a relatively steep plane of the environment. Moreover, a certain distance is maintained between the obstacles. The optimal path is more stable, and it meets the requirements of the algorithm for path planning. However, the path slightly increases the simulation time of the algorithm.

\subsection{Simulation 3}

Simulation 3 chooses diagonal points in the environment as the starting point and the target point, with the set start point $(1,18,800)$ and the goal point $(21,5,1500)$. The parameter setting of the algorithm simulation process still uses the values in Table 1. The experiment is conducted eight times. The planning path length of the conventional ACO and the IACO is shown in Table 6.

Table 6. Comparison of path length (unit: $\mathrm{km}$ )

\begin{tabular}{cccccccccc}
\hline & 1 time & 2 times & 3 times & 4 times & 5 times & 6 times & 7 times & 8 times & average \\
\hline ACO & 33.3286 & 35.6225 & 36.0060 & 33.0330 & 33.1249 & 37.3286 & 35.2400 & 32.2071 & 34.4863 \\
\hline IACO & 29.7300 & 29.7248 & 29.7407 & 29.7300 & 29.7401 & 29.7191 & 29.7032 & 29.7243 & 29.7265 \\
\hline
\end{tabular}

In Experiment 3, the optimal path length of ACO planning is $32.2071 \mathrm{~km}$, and the optimal path length of IACO planning is $29.7032 \mathrm{~km}$. The simulation comparison charts are shown in Figure 12,Figure 13 and Figure 14.

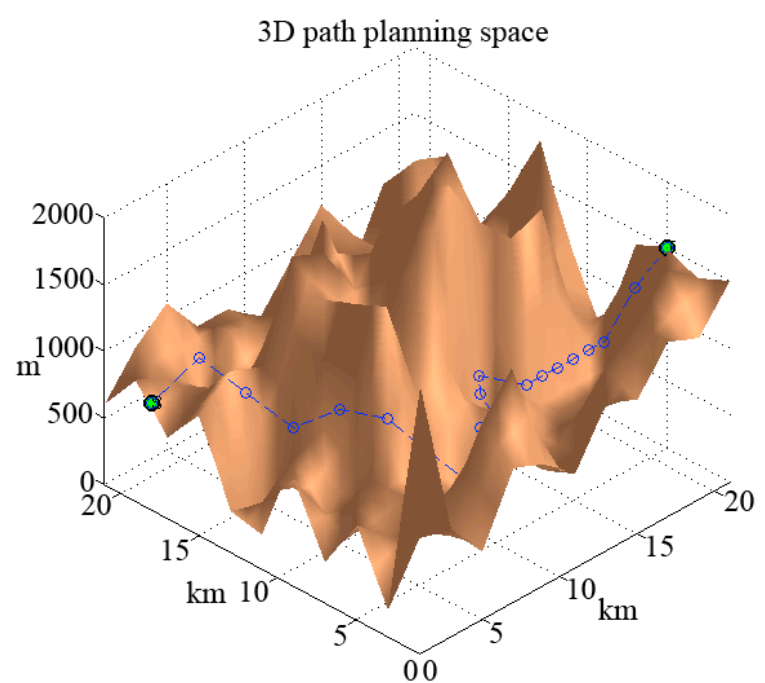

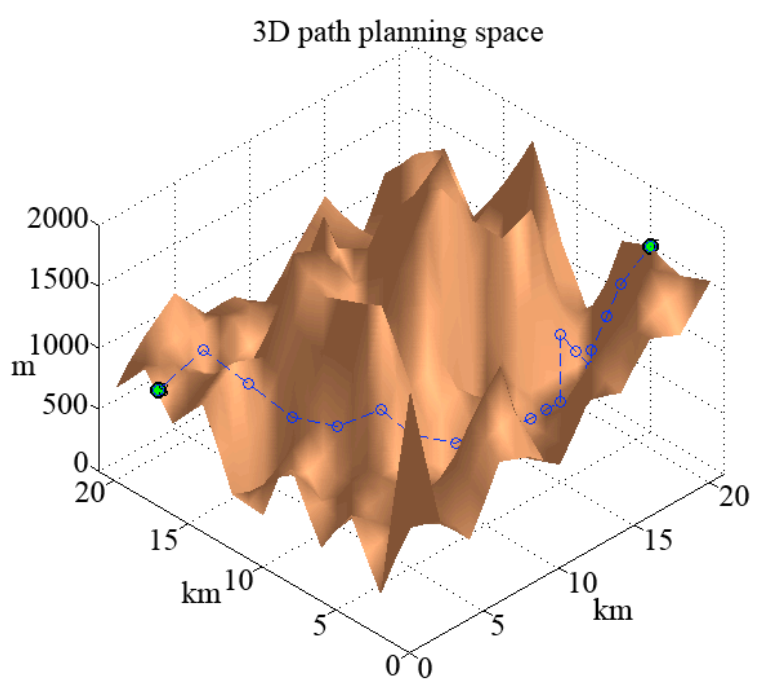

Fig. 12. Perspective diagram of optimal path with ACO and IACO planning 

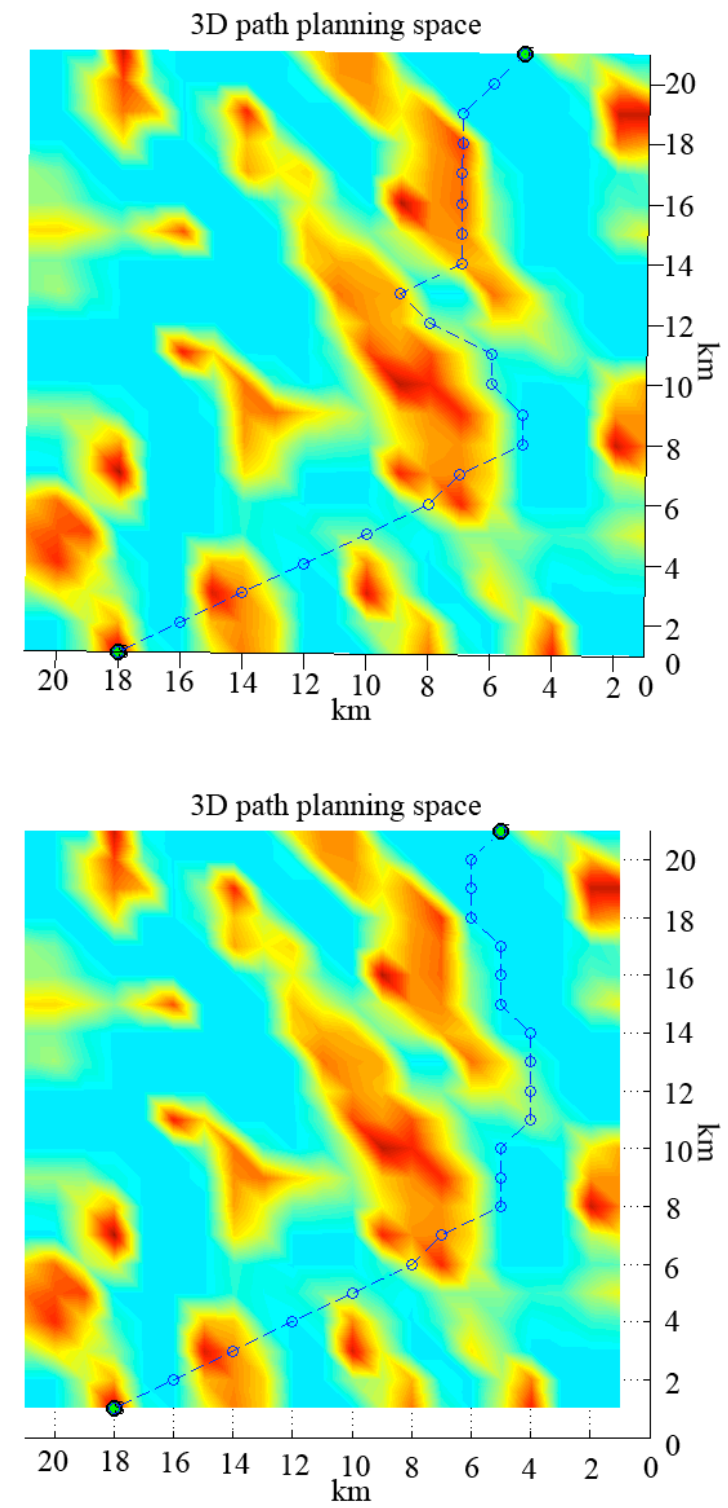

Fig. 13. Top view of optimal path with ACO and IACO planning

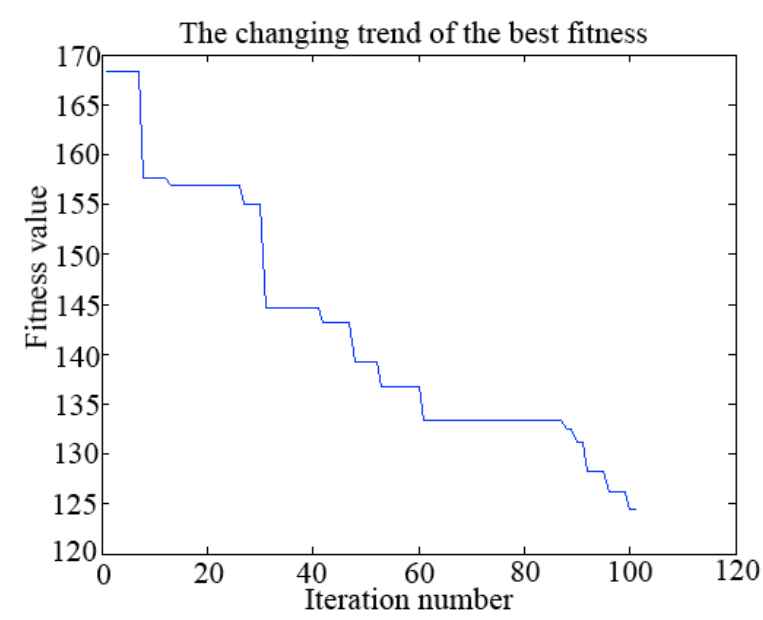

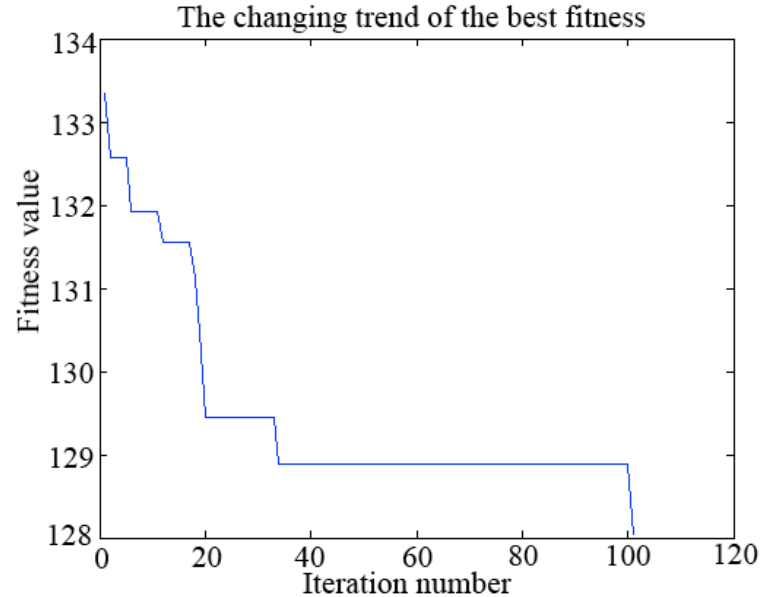

Fig. 14. Fitness variation diagram of ACO and IACO planning

In this simulation, the optimal path time comparison table of the ACO and IACO algorithm is shown in Table 7.

Table 7. Optimal path time comparison table

\begin{tabular}{l|l}
\hline & E_time(s) \\
\hline ACO & 4.945559 \\
IACO & 1.953487 \\
\hline
\end{tabular}

In Experiment 3, the simulation results show that the 3D average path length of IACO planning is much shorter than that of ACO in a relatively complex plane of the environment. In addition, the optimal path is more stable; the running time of the IACO algorithm to obtain the optimal path is shorter than that of the ACO.

The simulations also show that the optimization ability in a complex environment of the IACO algorithm is stronger than those of others.

\section{Conclusion and Future Work}

In this paper, the IACO algorithm with pheromone exclusion was proposed, and it was used to study the 3D path planning problem in complex underwater environments. To optimize the $3 \mathrm{D}$ path, we established the model of the $3 \mathrm{D}$ space in the underwater environment. The ant colony algorithm based on pheromone exclusion was proposed and applied to this algorithm for path planning in 3D space underwater. The objective was to find the optimal path between the start and goal points under three different environments. We presented the detailed process of the algorithm and compared the conventional ant colony algorithm and its improved version. The simulation results show that the algorithm proposed in this paper can improve search quality and accuracy. The output stability is good, especially in the complex plane environment. Several problems were also observed. For example, the searching time of the algorithm is extremely long and the planning path is not the global optimal path. Our future work aims to focus on how to speed up the convergence of the algorithm and how to improve the global optimization ability of the algorithm.

\section{Acknowledgements}

This study was partially supported by the Science and Technology Project of Guangdong Province of China (Grant No. 2014A020208087), the National Natural Science Foundation of China (Grant No. 61573157), the Fund of Natural Science Foundation of Guangdong Province of 
China (Grant No. S2013040015755), and the National Spark Technology Project (Grant Nos. 2014GA780051 and
2013GA780044). The authors are grateful to the anonymous reviewers for their useful comments and suggestions.

\section{References}

1. Chen, X., Kong, Y., Fang, X., and Wu, Q., “A fast two-stage ACO algorithm for robotic path planning", Neural Computing and Applications, 22(2),2013,pp.313-319.

2. Ghoseiri, K., Nadjari, B., "An ant colony optimization algorithm for the bi-objective shortest path problem", Applied Soft Computing, 10(4), 2010, pp.1237-1246.

3. Qi, Y., Wei, Z.Q., Yin, B., Fei, Y.D., Yu, Z.D., and Zhuang, X.D., "Enhanced ant colony algorithm for optimal path planning of mobile robot", Journal of Harbin Institute of Technology, 41(3), 2009, pp. 130-133.

4. Tan, G.Z., He, H., and Sloman, A., "Ant colony system algorithm for real-time globally optimal path planning of mobile robots", Acta Automatica Sinica, 33(3), 2007, pp.279-285.

5. Sun,M. X., Wang,Y.H., Wu,X.M., Feng,X.S., and Guan,Y.L., "The underwater robot path planning based on dynamic compensation", Robots, 15(2)1993,pp.8-12.

6. Carroll, K.P., McClaran, S.R., Nelson, E.L., Barnett, D.M., Friesen, D.K., and William, G.N., "AUV Path Planning: An A* Approach to Path Planning Width Consideration of Variable Vehicle Speeds and Multiple, Overlapping, Time Dependent Exclusion Zones", Proceedings of the Symposium on AUV Technology, 1992, pp.7984.

7. Hao, Y.L., Zhang, J.J., "AUV path planning in 3D seabed based on genetic algorithm”, China Engineering Science, 5(1), 2003, pp.5660.

8. Chen,S. M., Xie,J., Chen,W. D., Fang,J. H., "Three-dimensional path planning based on HPSO algorithm", Journal of Huazhong University of Science and Technology,2(41), 2013.pp.109-113.

9. Blum, C., "Ant colony optimization: Introduction and recent trends", Physics of Life reviews, 2(4), 2005, pp.353-373.
10. Narasimha, K. V., Kivelevitch, E., Sharma, B., and Kumar, M., "An ant colony optimization technique for solving min-max multidepot vehicle routing problem", Swarm and Evolutionary Computation, 13, 2013, pp.63-73.

11. Reed, M., Yiannakou, A., and Evering, R., "An ant colony algorithm for the multi-compartment vehicle routing problem", Applied Soft Computing, 15, 2014, pp.169-176.

12. Yang, X.S., Nature-Inspired Optimization Algorithms, England, London, Elsevier, 2014, pp.30-62.

13. Liu, L.Q., Dai, Y.T., "3D Space Path Planning of Complex Environmental Underwater Vehicle", International Joint Conference on Computational Sciences and Optimization, 2, 2009, pp.204-209.

14. $\mathrm{Hu}, \mathrm{H} .$, Research of robot $3 d$ path planning technology based on improved ant colony algorithm, Zhejiang: Zhejiang Normal University, 2012.

15. Xiao, Q. K., Wang, G., Luo.Y. C., "Three dimensional path planning for partial differential elevation map of ant colony algorithm", Systems engineering and electronics, 12(32), 2014, pp.1-13.

16. Liu, L.Q., Yu, F., Dai, Y.T., "3D Space Path Planning of Underwater Vehicle based on ant colony algorithm", Journal of system simulation, 20(14), 2008, pp.3712-3716.

17. Varela, G. N., Sinclair, M. C., "Ant Colony Optimization for Virtual-Wavelength-Path Routing and Wavelength Allocation", In Proceedings of the IEEE Congress on Evolutionary Computation, 3, 1999, pp.1809-1816.

18. Dorigo, M., Gambardella, L. M., "Ant Colony System: A Cooperative Learning Approach to the Traveling Salesman Problem", IEEE Transactions on Evolutionary Computation, 1(1), 1997, pp.53-66. 